\title{
Disruption of size discrimination in squirrel monkeys (Saimiri sciureus) by LSD-25'
}

\author{
LAWRENCE G. SHARPE ${ }^{2}$, LEON S. OTIS AND RONALD J. SCHUSTERMAN
}

STANFORD RESEARCH INSTITUTE

A "difficult" size discrimination (ratio 1.12:1) was disrupted in four of five squirrel monkeys by 10 to $40 \mu \mathrm{g} / \mathrm{kg}$ LSD-25 whereas an "easy" discrimination (ratio 1.96:1) was relatively unaffected. Disruption lasted from 4-6 months postinjection in two of the monkeys. Neither the difficult nor the easy discrimination was affected in three monkeys by up to $100 \mu \mathrm{g} / \mathrm{kg}$ BOL-148.

Fuster (1959) reported that 2 to $8 \mu \mathrm{g} / \mathrm{kg} \mathrm{LSD-25}$ administered to rhesus monkeys disrupted a learned discrimination between a cone and a pyramid presented tachistoscopically for $20 \mathrm{msec}$. He concluded that LSD-25 disrupted the animals' ability to respond to "the attention-requiring tachistoscopic task." Since adequate performance in a tachistoscopic task depends upon accuracy of visual discriminations and quickness of response, as well as attentiveness, this interpretation is ambiguous. In our research we sought to assess the effects of LSD-25 on visual discrimination performance by allowing ample opportunity for the animal to attend to the relevant stimuli and by de-emphasizing speed of motor performance. We did this by permitting our Ss to view the discriminanda for $4 \mathrm{sec}$. before they could make a response.

Method

Eight adult male squirrel monkeys, on a 20-hr. food deprivation schedule, were trained in a WGTA to displace one of two vertically-presented circular black plastic discs, differing only in size, in order to obtain a $1 / 16$ piece of grape concealed in a small well under one of the stimulus objects. Each trial began when a tray containing the stimulus objects was slowly pushed to the front of the monkey cage. Approximately $4 \mathrm{sec}$. elapsed before the tray was within reach of the monkey, and the trial terminated once object displacement occurred. Position of the stimulus objects was randomly varied.

The experiment consisted of five main phases: a training phase, a threshold phase, a drug phase, a postdrug phase, and a final threshold phase. In the training phase, four animals were trained to choose the smaller of two circular discs (size difference ratio $=3.84: 1$ ), and the other four animals were trained to choose the larger until a criterion of 45 correct responses out of 50 daily trials had been reached. ${ }^{3}$ This training was then twice replicated at successively smaller size difference ratios (2.19:1 and 1.54:1).

The threshold phase consisted of obtaining size threshold curves for each animal by the psychophysical method of constant stimuli. The purpose of this phase was to determine, for each animal, size ratios that were "difficult" (70-80\% correct responses) and "ea sy" (90-100\% correct responses) to discriminate. Twenty trials at each of four size ratios (shown on the abscissa of Fig. 2) were presented at each daily session. Ten sessions were run, totaling 200 trials for each size ratio. Only the easy and the difficult levels of discrimination were used during the drug phase. Testing began $30 \mathrm{~min}$. after injection and each session consisted of 30 trials each at the easy and difficult discrimination levels.

During the drug phase, intraperitoneal injections of LSD-25 were administered to five of the eight animals; the other three received BOL-148, a 2-brom derivative of LSD-25. Performance on the easy and difficult discriminations was first tested for 30 trials/ day for five days after a daily placebo injection of distilled water; then all animals were retested for 30 trials/day for five days following administration of $10 \mu \mathrm{g} / \mathrm{kg}$ each day of either LSD-25 or BOL-148. The dose level for each drug was increased in $10 \mu \mathrm{g} / \mathrm{kg}$ increments each successive week, until a change in performance of at least 15 percent had occurred over the week's testing on the easy or the difficult discrimination in relation to placebo performance. Then the postdrug phase, identical in procedure to the drug phase, consisting of two more five-day sessions, was initiated; one, a final placebo test session and the other a non-injected control test session. This was to determine whether LSD-25 may have had any residual effects on visual discrimination after the drug had been discontinued. The final threshold phase was identical in all respects to the first.

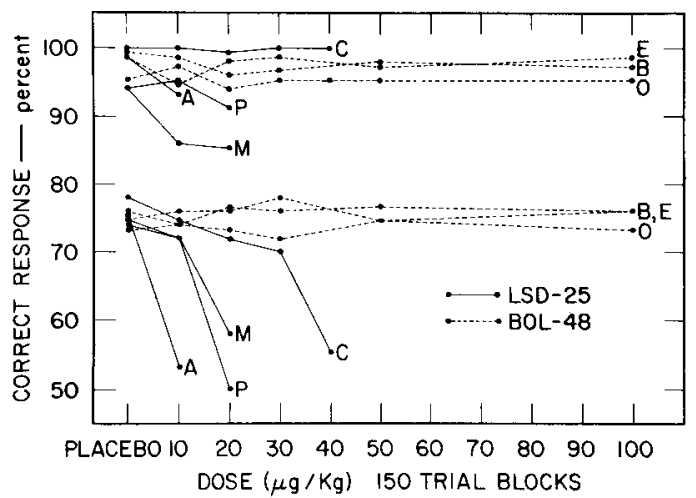

Fig. 1. Percent correct responses on the "easy" and "difficult" discriminations after placebo and various doses of either LSD-25 or BOL-148. The letters refer to individual animals. The upper family of curves represent performance on the "easy" discrimination and the lower, on the "difficult" discriminations. 


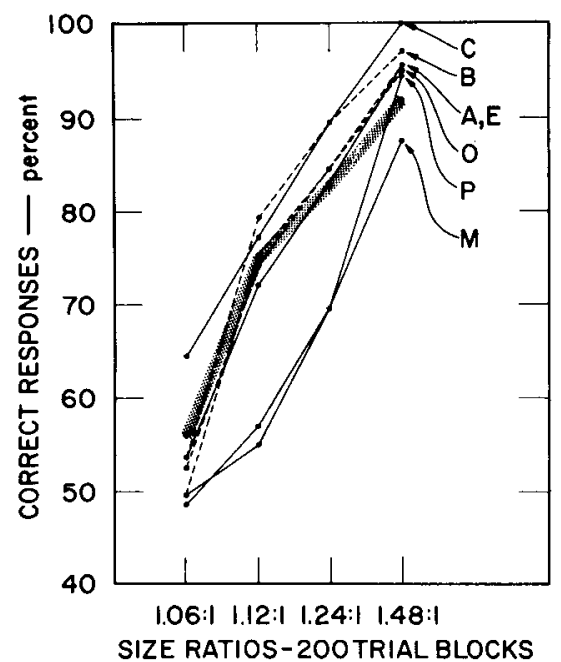

Fig. 2. Final (postinjection) size threshold curves for individual animals. Dotted and solid lines represent curves for animals that had received BOL-148 and LSD-25, respectively, during the drug phase. The shaded line represents the mean threshold curve for all animals during the initial threshold testing phase.

\section{Results and Discussion}

Only data for seven animals is presented; one animal (N) failed to reach our performance criterion of $70-80 \%$ correct responses for the $1.12: 1$ size ratio (difficult discrimination) and $90-100 \%$ correct responses for the 1.48:1 size ratio (easy discrimination). ${ }^{4}$

Performance on the easy and difficult discriminations for placebo and various drug doses are shown in Fig. 1. The only significant changes in performance were an average drop of 22.6 percent on the difficult discrimination in four animals given LSD-25 in amounts ranging from 10 to $40 \mu \mathrm{g} / \mathrm{kg}$. These changes were statistically significant for each $S$ when compared with placebo performances $(p \leq .001)$. BOL-148 in amounts up to $100 \mu \mathrm{g} / \mathrm{kg}$ produced no effect on discrimination performance.

Figure 2 compares the performance of Ss during the final threshold phase with the mean performance achieved during the initial threshold phase (shaded line). Unlike animals $A$ and $C$, which regained their previous level of threshold performance, two animals, $M$ and $P$, did not match their previous threshold curve. All three BOL-148 animals (dotted lines) matched their previous level of threshold performance.

These results show that LSD-25, in amounts of 10 to $40 \mu \mathrm{g} / \mathrm{kg}$, disrupted performance on difficult discrimination tasks, whereas relatively easy discriminations were much less affected ( 5 to 10 percent in 3 of 4 animals showing a decrement).

The effects of LSD-25 proved to be variable, both in terms of the dose level needed to impair performance and the time needed to recover from this effect. For example, animal $\mathrm{C}$ appeared highly resistant to LSD-25 when compared with animal A; yet both animals eventually regained their previous level of performance within a 2 to 3 week period after the last LSD-25 injection. The opposite was true for animals $\mathbf{P}$ and $M$, since recovery to predrug levels of performance did not occur until approximately 4 months and 6 months, respectively, after the last drug injection; all animals were retested at least once weekly after the final threshold testing phase. Variable effects of LSD-25 between Ss, especially when animals of the higher phyla, such as the primates, are used have been reported by others (Kornetsky et al, 1957; Jarvik \& Chorover, 1960). Differences in the doses of LSD-25 required to affect discrimination ability in our animal Ss may reflect differences between our Ss in ability to detoxify chronically administered LSD-25.

The present results indicate that LSD-25 may lessen the organism's ability to make fine, subtle, or difficult size discriminations, whereas easy discriminations remain relatively unimpaired. Recent work has suggested that animals repeatedly shift their attention from the relevant dimension to irrelevant stimuli when a discrimination becomes difficult, even when ample time is permitted for the animal to respond (Schusterman, 1966). We suspect that LSD-25 may have facilitated such attentional shifts to irrelevant stimuli during the presentation of the difficult discrimination.

\section{References}

Evarts, E. V. Neurophysiological correlates of pharmacologically induced behavior disturbances. In: The brain and human behavior. Proceedings of ARNMD, Vol. XXXVI. Baltimore: Williams \& Wilkins, 1958. Pp. 347-380.

Fuster, J.M. Lysergic acid and its effects on visual discrimination in monkeys. J. nerv. ment. Dis., 1959, 129, 252-256.

Jarvik, M. E., \& Chorover, S. Impairment by lysergic acid diethylamide of accuracy in performance of a delayed alternation test in monkeys. Psychopharmacologia, 1960, 1, 221-230.

Kornetsky, C., Humphries, O., \& Evarts, E. V. A comparison of the psychological effects of certain centrally acting drugs in man. Arch. Neurol. Psychiat., 1957, 77, 318-324.

Schusterman, R. J. Serial discrimination-reversal learning with and without errors by the California sea lion. J. Exptl. Anal. Behav., 1966, 9, 593-600.

\section{Notes}

1. Supported by Contract Nonr-2993 (00) between the Office of Naval Research and Stanford Research Institute.

2. Present address: Department of Psychology, Purdue University, Lafayette, Indiana.

3 . The discs used in the several phases of this experiment varied in size from $6.58 \mathrm{~cm}^{2}$ to $25.28 \mathrm{~cm}^{2}$.

4. Animal $\mathrm{N}$ was carried through the experiment even though this animal stabilized at a low level of discrimination performance. Animal N's performance on the easy discrimination continued to improve during the drug phase up to a dose of $70 \mu \mathrm{g} / \mathrm{kg}$, (the highest dose used with this animal), but no improvement occurred on the on the difficult discrimination; accuracy remained at approximately chance levels throughout the whole experiment. Improved performance of this animal on the easy discrimination fails to support Evart's (1958) suggestion that LSD-25 selectively disrupts habits that are in the process of being learned, leaving well-established habits relatively unaffected. 